The above cases referred to should be sufficient to urge orthodontists to give more consideration to these extreme oral malformations. Unfortunately orthodontists pay little or no attention to the benefits that can be derived through surgery, while oral surgeons give little or no consideration to the science of orthodontics. These two specialties are so closley related that one cannot progress without the knowledge of the other in successfully treating many of these pronounced oral malformations.

\title{
THE RELATION OF ORTHODONTIA TO DENTISTRY.*
}

\author{
By Frederick Bogue Noyes, B.S., D.D.S. \\ Professor of Orthodontia and Histologì, University of Illinois, College of Dentistry, Chicago.
}

THE present is an age of specialization. Knowledge and skill have made such rapid advancement that it is impossible for any man to compass the whole range of knowledge or excel in many fields of execution. The engineer must decide whether he will follow some line of machine construction, factory construction, the development of hydraulic power, electrical power, the reclamation of land, or some other field of constructive endeavor. The physician must choose the study of the eye, the ear, the nose and throat, the stomach, the genitourinary tract, or some other field. So also the dentist must choose between the treatment of the diseases of the hard tissues of the teeth, the treatment of the soft tissues, the construction of artificial dentures, the correction of malocclusion, or the extraction of teeth, for he cannot hope to excel in all of them.

This development of specialization demands a foundation of broad knowledge and skill, and their application in a limited field. No matter how limited the field of application, knowledge of the fundamental sciences must be broad. No man can hope to push forward the knowledge of the bacteriology of the mouth without a good foundation in general bacteriology. No one can be a good oral surgeon without a knowledge of the fundamental principles of general surgery, and training and experience in its technic and practice.

To rise out of mediocrity to a position of distinction, today requires vastly greater effort and attainment than a century ago. This is true because every specialized field is drawing from every department of science with increasing efficiency, applying the fullest development of each science to the accomplishment of its own end, and every department of science has made such rapid progress in the last few ycars that it would be impossible for any one to excel in them all. It is a mistake, however, to suppose that every new discovery in a department of science is revolutionary and destroys the work that has preceded it; and one who has once mastered the fundamentals of science, may keep in touch with its advancement with comparatively little effort while he would not be at all in a position to carry on a new work in this field, to be in the vanguard pushing that line still further into *Read before the Illinois State Dental Society, May, 1915. 
the unknown. There always has been and there always will be two types of minds: the encyclopedic and the logical types, and each has its sphere in the development of any profession or science. The man with the encyclopedic type of mind knows and remembers what everyone else has said and thought on his particular subject. He can tell you when this article was published by that man and another article by another man and just the point of view of each individual. But when he attempts the investigation of a new field he is very apt in generalizations to miss the most important points and just as likely to emphasize the unimportant details and miss entirely the one central thing. In contrast to this type, the logical type compares accurately, sees clearly all individual facts, and recognizes the generalizations that can be drawn from them. He thinks clearly and works out an individual philosophy or interpretation of the facts to the best of his ability and in so far as he goes, truly, but he may be entirely ignorant of what any one else's opinion has been in the same field. To a certain extent these two types are contradictory but they are not necessarily so. To draw a comparison from current military tactics, the encyclopedic type may be compared to the general who, conscious and posted continually as to every point and as to what is going on throughout the entire line, is not in direct contact with the men at all, and the officcrs who, entirely ignorant of what is going on at any other point on the battle front, are engaged only in pushing forward the attack, at their own particular points. In the development of a profession and the training of men for the pursuit of highly specialized fields, the broadness of the preliminary training should be emphasized to counteract the narrowing tendency of the intensive following of a limited field. The training should be planned not simply to develop the highest degree of efficiency, and attain the most rapid advancement in a special field, but to keep the specialty in touch with the general profession and co-ordinate it with other specialties for the benefit of humanity.

The development of specialization as it is today, has brought about the interdependence of the general practitioner of dentistry and the various specialties. And for the first time, it has brought the dentist into the same relationship to the diagnostician and the internal medicine man as are the other specialties in medicine. Now, for the first time, the physician is looking for and expecting the same co-operation from the dentist that he has been accustomed to look for and receive from the oculist, the rhinologist, and other specialists. A physician, finding a patient suffering from some condition which he knows to be caused by some local infection or inflammation refers the patient to the dentist for a report on his oral condition, just as he refers the patient to the rhinologist. for a report on the mucous membrane of the nose or the condition of the tonsils. The physician, in that case, is not concerned with the teeth as a masticating apparatus, but he wishes to know if there is any pathologic condition present, and if so, an intelligent report as to its character and extent, that he may have some judgment as to its relation to the general pathologic condition. If the dentist replies upon superficial examination that he finds the mouth apparently normal, as a masticatory apparatus, without a detailed report as to the presence or absence of inflammation of the gingivæ, the presence or absence of suppurative pockets on the sides of the roots of teeth, the presence or absence of sup- 
purative conditions about the apices of tooth roots, and their extent and character, and certainly if he replies without a radiographic examination of both arches to reveal the possible existence of suppuration within the substance of the bone, he fails utterly from the standpoint of the physician. What the physician wished was the opinion of an expert in the given field's pathology that he could use as a basis for the organization of his campaign for the health and usefulness of the individual, and too of ten he is unable to get such reports. In such conditions, too, the specialist should remember the general systemic conditions which are present should govern him as factors in determining the character of the treatment to be used for the local condition. In a local suppurative condition, the treatment for a patient not suffering from any systemic condition might be quite justifiable, which would not be justifiable at all for a patient showing manifestations of some grave general condition.

Just as the physican looks to the dentist for expert advice, so one specialist looks to another for the same character of expert judgment. The oculist may call upon the dentist for the same character of advice and treatment as is sought by the intern. The rhinologist, having removed a nasal obstruction and created normal conditions in the nasal pharynx, knows that normal respiration will be impossible until the malocclusion is corrected so that the normal respiratory functions can be carried out. The orthodontist correcting a malocclusion in which teeth or the cusps of teeth have been lost or destroyed sends the patient to the general practitioner, for the restoration of the lost teeth or cusps, knowing that unless this is done the preservation of normal occlusion will be impossible. So the specialist looks upon the socalled general practitioner as a specialist in his department.

To show you the importance of this particular matter, a concrete example is cited. In the correction of malocclusion in the writer's practice, an upper first molar was found in lingual occlusion. It had never been perfectly formed and caries had destroyed all its cusps, an amalgam filling had been made which was as flat as a board. The patient was sent to the general practitioner to have this replaced by a gold inlay that would restore the normal-sized crown and the form of the occlusal surfaces. The patient returned with the gold inlay, beautiful from a mechanical standpoint, with perfect margins, but with cusps that resembled no tooth in heaven above or earth beneath, and from the standpoint of normal occlusion, utterly functionless. With such an occlusal surface, there was no hope of maintaining the normal occlusal relations of that tooth.

I have dwelt upon the development of interdependence among the specialties and of the disappointment that is too often experienced, as the basis of an appeal for better types of specialists in dentistry. In medicine a man would not think of attempting to practice as a specialist without going to those places where material is abundant and devoting time, energy, and money to study under the men of the greatest knowledge and skill, developing there his own knowledge and experience in public clinics and hospitals under the direction of able men, before attempting to conduct private practice of a specialty. In medicine the means of developing and training specialists is a method well developed all over the world, but up to the present time there has been nothing corresponding to it in dentistry. 
At present the partisan, I had almost said "charlatan," who travels about the country exploiting some special method of treatment, often backed up by some commercial house, claiming to equip men for the practice of some specialty, are a disgrace to the dental profession, and they exist chiefly, I believe, because educational institutions have failed to offer opportunities for special study and training and because the dental profession has not yet organized hospitals and infirmaries for the care of the poor of the communities who are unable, for financial reasons, to obtain dental service, and which should be the places for special training. There is just as much reason for providing dental service for the poor of any locality as there is for providing hospital service. Every state and public institution should maintain dental attendance as they do medical attendance, and in the future, I believe it will be the function of the state dental societies to organize this service so that it will be the means of training specialists in dentistry, just as corresponding institutions are the means of training specialists in medicine. In medicine, the hospital and public infirmary has grown out of the medical school and the university. In the same way, I believe that the first step is the development in the universities and dental schools of adequate means for training specialists in connection with hospital and infirmary service. Such a development, however, requires the proper point of view of the profession in general. In the past, dentistry has been too ready to accept the short course of a few weeks as a training for special practice and it is a grave question whether men would devote a year's time in the pyorrhea service or the orthodontia service in a dental hospital as men devote a year or two to an intern service in such an institution as the Cook County Hospital, in the eye, ear or surgical service. But I believe that if such fair training could be offered under adequate supervision coupled with thorough courses in the allied sciences, there would be many young men of great ability who would avail themselves of the opportunity.

Orthodontia is a specialty of dentistry, in just about the same sense that dentistry is a specialty of medicine. For the training for general practice of orthodontia does not train a man for the practice of orthodontia any more than the training for medicine equips a man for the practice of dentistry. The good of both orthodontia and dentistry depends upon the admission of this relationship. As evidence of the great development of orthodontia in the last few years, one had only to notice the great number of children wearing appliances and the large proportion of space in dental literature devoted to the subject. But as I view it, there is bound to be reaction. For too large a proportion of the men practicing it are utterly. untrained either in the theory or the technic of orthodontia. The result must be an enormous crop of failures. I believe that eventually every fairsized city will have its orthodontist, supported by the dentists, just as the present time every fair-sized city has its surgeons supported by the general practitioners. But this condition for orthodontia can never come until there are a sufficient number of institutions presenting courses which will adequately train men for practice.

The development of transportation facilities in the interurban electric railroad, and the enormous increase in the use of the automobile, will favor the development of specialists in the smaller cities, for it increases the area 
from which specialists may draw patients, but in addition to these favorable conditions, there is needed the growth of a feeling of co-operation between the individuals of the profession in order to maintain a specialist, except in the largest cities. Men in a given locality must allow the one among them with the greatest ability and liking for a given specialty to go where he can receive the special training and practice necessary for the practice of that specialty, and then if possible refer all such cases in that locality to him for treatment. Such a method would be greatly to the advantage not only of the community but of the profession.

\title{
THE GAUSE AND EFFEGT OF ORAL DEFORMITIES.*
}

\author{
BY DR. A. C. GIFFord, OShKosh, Wis.
}

WE know by this time that dentisiry has begun to broaden its field. There are now specialists in mc: every line. The most important, exact and pronounced specialty is that which has to do with the formation of the jaws of man. To be able to mould a deformed jaw into a perfect one is an exact science. Orthodontics is that exact science. When we realize how great is the need of normal position of the teeth, normal relation of the arches and harmony in the size of the arches, then and only then can we realize the great field of orthodontia.

The jaws at birth, unless there be congenital disturbances, are in the proper relation to the face and head and to each other. As there is nothing to interfere with the eruption of the temporary teeth, they will usually erupt in their proper position if the child is of normal health and develops normally. There are cases occasionally where there is a mesial or distal relation of the lower arch, but these cases are not frequent.

It is not possible, in my mind, to tell the cause of every case of malocclusion even though we do have a definite history, but we can systematize our knowledge in such a way that the average case will give us a clear vision of the cause.

- W/Then we speak of inherited malocclusion we think of some relative having the same class of deformity as the case under consideration. May not this be a case of the child living in the same environment as did the relative or may it not be congenital? There are able men that claim that there are no cases or inherited malocclusion. In the past few years these men have had occasion to treat and study the different forms of malocclusion to a great extent and have divided causes into either local or general. We must confess that from the standpoint of biology, embryology, anatomy and histology there is no evidence of malocclusion ever being inherited. Each individual germ-cell possesses a certain amount of chromatin that presides over inheritance and we have no evidence that malocclusion is impressed upon the chromatin of that cell. We hear such things as this child inherited the large teeth of the father and the small jaw of the mother. How can this

* Read before the Central Wisconsin Dental Society and the Oshkosh Dental Society, October 12, 1915. 